\title{
The multi-objective railway timetable rescheduling problem
}

\author{
Stefan Binder * $\quad$ Yousef Maknoon * ${ }^{*} \quad$ Michel Bierlaire * \\ May 30, 2016
}

Report TRANSP-OR 160530

Transport and Mobility Laboratory

École Polytechnique Fédérale de Lausanne

transp-or.epfl.ch

${ }^{*}$ Transport and Mobility Laboratory (TRANSP-OR), School of Architecture, Civil and Environmental Engineering (ENAC), École Polytechnique Fédérale de Lausanne (EPFL), CH-1015 Lausanne, Switzerland, \{s.binder, yousef.maknoon, michel.bierlaire\} @epfl.ch 


\begin{abstract}
Unexpected disruptions occur for many reasons in railway networks and cause delays, cancellations, and, eventually, passenger inconvenience. This research focuses on the railway timetable rescheduling problem from a macroscopic point of view in case of large disruptions. The originality of our approach is to integrate three objectives to generate a disposition timetable: the passenger satisfaction, the operational costs and the deviation from the undisrupted timetable. We formulate the problem as an Integer Linear Program that optimizes the first objective and includes $\varepsilon$-constraints for the two other ones. By solving the problem for different values of $\varepsilon$, the three-dimensional Pareto frontier can be explored to understand the trade-offs among the three objectives. The model includes measures such as cancelling, delaying or rerouting the trains of the undisrupted timetable, as well as scheduling emergency trains. Furthermore, passenger flows are adapted dynamically to the new timetable. Computational experiments are performed on a realistic case study based on a heavily used part of the Dutch railway network. The model is able to find optimal solutions in reasonable computational times. The results provide evidence that adopting a demand-oriented approach for the management of disruptions not only is possible, but may lead to significant improvement in passenger satisfaction, associated with a low operational cost of the disposition timetable.
\end{abstract}

Keywords: railway timetable rescheduling, passenger satisfaction, multi-objective, Pareto frontier, ILP 


\section{Introduction}

Major disruptions, such as the unavailability of railway tracks due to unexpected events (e.g., rolling stock breakdown, adverse weather conditions), occur frequently in railway networks. For instance, Jespersen-Groth et al. (2009) report approximately 22 infrastructure-related disruptions per day, with an average duration of 1.7 hours, on the Dutch railway network during the first half of 2006 . These events cause train delays and cancellations, which lead to passenger dissatisfaction. Due to the disruption, passengers using the railway network have longer travel times and reach their destination later than expected. Also, passengers can experience even larger delays if they miss a connection.

The deregulation of the European railway market in recent years has thus pushed railway operators to focus on the level of service provided to customers. Now more than ever, it is crucial for railway operating companies to outperform the competition in terms of passenger satisfaction, measured with indicators such as punctuality or reliability. Providing an adequate response to disruptions is a necessary step in this direction. Also, avoiding the cost of increasingly common "compensation payments" to passengers suffering from large delays is a strong incentive for railway operators.

The reasons outlined above call for a framework that is able to quantify the response to disruptions and evaluate trade-offs between the different stakeholders when designing a disposition timetable. Due to its complexity, the recovery problem is usually broken up into three consecutive phases: timetable rescheduling, rolling stock rescheduling and crew rescheduling. In this paper, we focus on timetable rescheduling. The timetable provided by our framework can then be used as an input for the second and third phases of the recovery problem, which is hard to solve in an integrated way.

In this paper, we consider major disruptions causing the unavailability of one (or more) track(s) for a known time period. We therefore look at the rescheduling problem from a macroscopic point of view, disregarding details such as track assignments in stations or signalling. Our framework generates a so-called disposition timetable, which is conflict-free in terms of operational constraints (e.g., no two trains can be scheduled on the same resource at the same time) and as convenient as possible for the passengers. When constructing a disposition timetable, the objective of the railway operator is to minimize the operational costs, while the aim of the passengers is to receive the best possible level of service. The two goals are usually incompatible: the best possible service for the passengers may also be the most expensive option for the operator. This inadequacy is the key motivation for our work: constructing disposition timetables that take into account passenger satisfaction, while keeping operational costs low. Furthermore, we consider the deviation from the undisrupted timetable as a common objective to be minimized by both passengers and the operator.

The main contribution of this paper is to propose an Integer Linear Programming formulation for the multi-objective railway timetable rescheduling problem in case of a major disruption. It takes into account the passenger satisfaction and imposes upper bounds on the operational cost and on the deviation from the undisrupted timetable. In contrast to the existing literature, our formulation allows total flexibility for the timetable rescheduling: a train can be delayed, totally or partially cancelled or rerouted through another part of the network, and emergency trains can be scheduled. In addition, passenger flows are adapted dynamically to the disposition timetable, as the preferred path of a passenger in the undisrupted case might not be available anymore in the new timetable.

To the best of our knowledge, this is the first attempt to integrate three objectives in a single frame- 
work for railway timetable rescheduling. The timetables constructed by this approach are therefore results of the trade-off between the conflicting objectives. The exploration of the three-dimensional Pareto frontier allows to analyze this trade-off and to quantify the quality of the timetables according to the three objectives. We solve the problem optimally on a realistic case study and show that the passenger satisfaction can be significantly improved at relatively low additional operational costs.

The remainder of this paper is structured as follows. Section 2 reviews the current state of the scientific literature in the railway timetable rescheduling field. The problem is formally described in Section 3 and presented as an Integer Linear Program in Sections 4 and 5. Section 6 reports the results of the computational experiments on the case study. Finally, Section 7 concludes the paper and provides directions for further research.

\section{Literature review}

The literature review presented in this section focuses on recent contributions to the train timetable rescheduling problem. It is mainly based on the review paper on railway recovery models by Cacchiani et al. (2014), where publications are classified according to three main criteria, defined in Table 1. Based on this classification, we identify gaps where contributions can be made to the literature, hence justifying the relevance of our work.

Table 1: Criteria classifying the railway recovery literature.

\begin{tabular}{ll}
\hline Criterium & Description \\
\hline Disturbance & $\begin{array}{l}\text { Primary delay (i.e., a process taking longer than initially scheduled) that can be handled by rescheduling } \\
\text { the timetable only, without rescheduling the resource duties (such as crews and rolling stock) } \\
\text { (Relatively) large external incident strongly influencing the timetable and requiring resource duties to be } \\
\text { rescheduled as well }\end{array}$ \\
\hline Microscopic & $\begin{array}{l}\text { Very precise representation of the railway infrastructure (sometimes at the switch or track section level), in } \\
\text { order to compute detailed running times and headways between trains }\end{array}$ \\
Macroscopic & $\begin{array}{l}\text { High-level representation of a railway infrastructure, considering only stations and tracks (details such as } \\
\text { signals or track sections are ignored) }\end{array}$ \\
\hline Operations-centric & $\begin{array}{l}\text { Focus on minimizing negative effects related to railway companies, such as delays or the number of can- } \\
\text { celled trains }\end{array}$ \\
Passenger-centric & $\begin{array}{l}\text { Focus on minimizing negative effects related to passengers, such as total travel time or number of connec- } \\
\text { tions }\end{array}$ \\
\hline
\end{tabular}

The thorough review of railway recovery models presented in Cacchiani et al. (2014) shows that the major part of the recent scientific literature deals with disturbances rather than disruptions. Further, in most papers, the railway network is represented at the microscopic rather than at the macroscopic level. Most papers also have an operations-centric approach to railway timetable rescheduling, instead of a passenger-centric view. The literature reviewed in this section focuses on the works most relevant to us, dealing with disruptions at a macroscopic level. First, operations-centric models are presented. Then, passenger-centric works are reviewed and the differences with the present work are pointed out. 


\subsection{Operations-centric railway disruption management}

Brucker et al. (2002) consider the problem of rescheduling trains in case of a track closure due to construction works on a double-track line. A local search heuristic that minimizes lateness is presented and tested on a real world instance. Corman, D'Ariano, Hansen, et al. (2011) consider a disruption on a double-track network, where some of the tracks become unavailable. The problem is split into separate dispatching areas, each of which are modelled individually. Finally, boundary constraints between the dispatching areas guarantee that the local solutions are globally feasible. This methodology is compared to a centralized approach, where general dispatching rules are imposed. For both approaches, the authors face increasing difficulty to obtain a feasible timetable for larger time horizons. Narayanaswami and Rangaraj (2013) develop a MILP model that resolves conflicts caused by a disruption that blocks part of a single bidirectional line. Trains can only meet and pass each other in the stations, and cannot be cancelled. Train movements are rescheduled in both directions of the line for a small artificial instance, with the objective of minimizing the total delay of all trains. Albrecht et al. (2013) consider the problem of disruptions due to track maintenance, arising when maintenance operations take longer than scheduled and thus force to cancel additional trains. A disposition timetable including track maintenance is constructed using a Problem Space Search meta-heuristic. The methodology is tested on a single track railway network in Australia. Corman, D'Ariano, Pacciarelli, et al. (2014) compare centralized and distributed procedures for train rescheduling, and propose heuristic algorithms to coordinate dispatching areas. The authors test their algorithms on a Dutch railway network with various traffic disturbances, including delays and blocked tracks.

Louwerse and Huisman (2014) consider the case of partial and complete blockades in case of a major disruption on a double track line. They develop a mixed-integer programming model to generate the disposition timetable. Two disruption measures are applied: train cancelling and train delaying. The objective is to minimize both of them. Schedule regularity constraints (e.g., operating approximately the same number of trains in each direction during a partial blockade) are included in the formulation in order to take the rolling stock problem into account implicitly. In case of a complete blockade, both sides of the disruption are considered independently (i.e., trains will reverse before the disrupted area but no coordination with the other side is considered). Veelenturf, Kidd, et al. (2015) extend the MILP model of Louwerse and Huisman (2014). The extended model is able to deal with a real-world railway network and includes the possibility to retime, reorder, cancel and reroute trains. The paper also considers the transition phases between the undisrupted timetable and the disposition timetable, as well as back to the original timetable when the disruption has ended. The model is tested on a part of the Dutch railway network, and in most cases the computational time is acceptable. Zhan et al. (2015) consider railway rescheduling on a high-speed line in case of a complete blockage. Due to the nature of the seat reservations, trains that have started their journey have to end in their final destination and cannot be rerouted or cancelled. The problem is formulated as a MILP with the same objective function as Louwerse and Huisman (2014). The model is tested on a real-world Chinese case study and is able to reduce the effects of the disruption on passenger service. 


\subsection{Passenger-centric railway disruption management}

Cadarso et al. (2013) develop an integrated optimization model for timetable and rolling stock rescheduling that accounts for dynamic passenger demand. The problem is solved in two iterative steps. First, the anticipated disrupted demand is computed using a logit model. As demand figures are estimated before the timetable is adjusted, they are based on line frequencies in an anticipated disposition timetable, rather than on actual arrival and departure times. In the second step, the timetabling and rolling stock rescheduling problem is formulated and solved as a MILP model, subject to the anticipated demand calculated in the first step. Recovery strategies include cancelling existing train services or scheduling extra ones. However, the possibility of retiming existing trains is not considered. Computational experiments are performed on the regional rapid transit network of Madrid. Veelenturf, Kroon, et al. (2014) also integrate the rescheduling of rolling stock and timetable in disruption management. Timetable decisions are limited to additional stops of trains at stations where they normally would not call. The fact that passengers will adapt their path to the new schedule is taken into account in a heuristic iterative framework: after each generation of a new schedule, passenger flows are simulated to evaluate the service from the passenger's point of view. Kroon et al. (2015) present a mathematical model and an iterative heuristic to solve the real-time rolling stock rescheduling problem with dynamic passenger flows. The rescheduled timetable is used as an input in their formulation. The model minimizes a combination of system-related costs (such as penalties for the modification of rolling stock compositions) and service-related costs that express the effect of train capacities on the total passenger delay. Computational results are reported on problem instances constructed from the Netherlands Railways network.

The delay management problem, initially introduced by Schöbel (2001), determines which passenger connections should be maintained in case of a delayed feeder train. Many extensions of the original model have been proposed (see, e.g., Schachtebeck and Schöbel, 2010; Schöbel, 2009). Two that are of particular interest to us are Dollevoet et al. (2012) and Corman, D'Ariano, et al. (2016). In the former, passengers can reroute themselves in the network if they miss a connection (in earlier models it was assumed that a passenger who misses a train waits for an entire cycle). In the latter, the authors integrate the microscopic representation of railway operations and the passenger perspective of the delay management problem.

\subsection{Contributions}

The three first papers presented in the previous section introduce a heuristic iterative framework to consider passenger flows during disruptions. In contrast, our model integrates the passenger travel choices with the timetable rescheduling model in order to generate convenient timetables for the passengers. In this sense, our work can be seen as an integrated version of these papers. Furthermore, we consider explicitly interactions between demand and supplied capacity, which Cadarso et al. (2013) disregards. Our work also allows for more rescheduling possibilities (retiming and rerouting of trains) than Veelenturf, Kroon, et al. (2014). The delay management problem introduced by Schöbel (2001) is not considered explicitly in our work; however, by minimizing the generalized passenger travel time in the objective function we also minimize connection times.

The contribution of this paper is summarized as follows: 
- We address the timetable rescheduling problem as a tri-objective problem, with a special emphasis on minimizing passenger inconvenience.

- We propose an exact mathematical model that integrates both the timetable rescheduling problem and passenger routing.

- We allow total flexibility for the new timetable and define a measure that quantifies the deviation from the undisrupted timetable.

- We carry out computational experiments on a realistic case study and are able to solve the model to optimality for several instances.

\section{Problem description}

We present a multi-objective timetable rescheduling framework for disrupted railway networks. The operator and the passengers have different goals in railway operations. Passengers would prefer a direct train from their origin to their destination, arriving exactly at their desired arrival time. Considering the operational cost of this solution, it is obviously impossible to provide such a service to every passenger. Hence, a trade-off between these two objectives needs to be found. In case of disruptions in railway operations, we also need to take into account the deviation from the undisrupted timetable. Considering this "cost" is necessary in order to avoid solutions where the schedule of the entire network is overhauled because of a local disruption. This is beneficial both for the passengers and the operator. Also, once the disruption is resolved, it is easier and quicker to come back to the undisrupted timetable if the disposition timetable is not too different. Given these objectives, our approach determines which trains should be delayed, cancelled or re-routed through another part of the network. We also include the possibility of scheduling "emergency trains", situated in shunting yards near given stations.

In the passenger railway service, a timetable is defined as the set of arrival and departure times of every train at each of the stations where it stops. As we are dealing with disruptions, we consider each train individually, instead of train lines. By solving the model, one obtains a disposition timetable as well as the modified routings of the passengers through the network.

\subsection{Infrastructural model}

Time is discretized into $n+1$ time intervals of length $\tau$ and we introduce the set of time steps $H=\{0, \tau, 2 \tau, \ldots, n \tau\}$, where $n \tau$ is the considered planning horizon. We model the railway network at a macroscopic level. The infrastructure is represented by a set of stations $s \in S$ and a set of tracks $\mathrm{Q} \subseteq \mathrm{S} \times \mathrm{S}$ connecting the stations. A track $\left(s, s^{\prime}\right) \in \mathrm{Q}$ is an uninterrupted railway track linking $s$ to $s^{\prime}$ directly, without passing in any other station. Each station $s$ is characterized by its available platforms $p \in P_{S}$ and the presence or absence of a shunting yard. We denote by $S_{R} \subseteq S$ the subset of stations with a shunting yard, and by $R$ the set of shunting yards. Every shunting yard $r_{s} \in R$ is associated with exactly one station $s \in S_{R}$. 
We define two stations $s, s^{\prime} \in S$ to be neighbouring if $\left(s, s^{\prime}\right) \in Q$ and $\left(s^{\prime}, s\right) \in Q$. Between two neighbouring stations, the running time $t\left(s, s^{\prime}\right)$, in minutes, and the distance $d\left(s, s^{\prime}\right)$, in kilometers, are known and equal for all trains. Trains cannot switch tracks between stations and overtakings occur only within stations (i.e., a platform in a station can be reached from any incoming/outgoing track). Each track can be used in one direction at the time, or it can be assigned to opposite directions alternatively. A certain headway is respected if two consecutive trains are running in the same direction on the same track. In case the track is used in opposite directions, a set of conflicting movements is defined to ensure proper separation of the trains.

Two different groups of trains are considered: original trains and emergency trains. The set of original trains $k \in \mathrm{K}$ contains the trains that are operated in the undisrupted timetable. Their schedule is an input to the rescheduling model. The set of emergency trains $e \in E$ represents trains that are located in shunting yards, ready to be scheduled if needed. All trains begin and end their trip at a shunting yard and the number of emergency trains available in each shunting yard is given by $n_{r}$. We assume that all trains are homogeneous, with the same capacity q, defined as the maximal number of onboard passengers.

\subsection{Passenger travel choice model}

We assume that passengers form groups that share the same origin-destination pair and desired arrival time at destination. As the travel time is deterministic in our modeling framework, the groups can equivalently be characterized by the desired departure time. We adopt the latter representation in the following. A passenger group $g \in G$ is denoted by a triplet $\left(o_{g}, d_{g}, t_{g}\right)$, where $o_{g} \in S$ is the origin station, $d_{g} \in S$ the destination station, and $t_{g} \in H$ the desired departure time from the origin. The number of passengers in group $g$ is $n_{g}$. The model does not allow splitting of the groups (groups of size one can be considered in order to model passengers individually).

For every passenger group, we consider the set $\mathrm{P}_{\left\{\mathrm{o}_{g}, \mathrm{~d}_{g}\right\}}$ of all paths linking the origin station $\mathrm{o}_{g}$ to the destination station $d_{g}$. A path is a sequence of access, in-vehicle, waiting, transfer and egress movements (refer to Section 4 for a definition in terms of arcs in a space-time graph). We associate a utility function with every alternative (i.e., path) and assume that each passenger group chooses the one with the highest utility. The utility function of every alternative $i \in \mathrm{P}_{\left\{\mathrm{o}_{g}, \mathrm{~d}_{g}\right\}}$ for passenger group $\mathrm{g}$ depends on the following attributes (see Robenek et al., 2016):

- in-Vehicle Time $\left(\mathrm{VT}_{\mathrm{i}}\right)$ : time, in minutes, spent by the passenger group in one (or more) train(s) along the path,

- Waiting Time $\left(\mathrm{WT}_{\mathrm{i}}\right)$ : time, in minutes, spent by the passenger group waiting between two consecutive trains at a station along the path (does not consider the waiting time for the first train),

- Number of Transfers $\left(\mathrm{NT}_{\mathrm{i}}\right)$ : number of times the passenger group needs to change trains along the path,

- Early Departure $\left(\mathrm{ED}_{\mathrm{i}}=\max \left(0, \mathrm{t}_{\mathrm{g}}-\mathrm{t}\right)\right)$ : time difference, in minutes, between the desired $\left(\mathrm{t}_{\mathrm{g}}\right)$ and the actual $(t)$ departure time from origin $(t)$, if early, 
- Late Departure $\left(\operatorname{LD}_{i}=\max \left(0, t-t_{g}\right)\right)$ : time difference, in minutes, between the actual $(t)$ and the desired $\left(t_{g}\right)$ departure time from the origin, if late.

We assume that the price of the trip is equal among all the paths for a given origin-destination pair, so that it does not need to enter the utility function (utility theory only considers differences between alternatives). Based on the aforementioned description for a given passenger group $\mathrm{g}$, the utility of the alternative $i$ is defined as follows:

$$
V_{i}=-\left(V T_{i}+\beta_{1} \cdot W T_{i}+\beta_{2} \cdot N T_{i}+\beta_{3} \cdot E D_{i}+\beta_{4} \cdot L_{i}\right),
$$

where $\beta_{1}, \ldots, \beta_{4}$ are the relative weights of the attributes described above. This quantity is in minutes and expresses the generalized travel time of passenger group $g$ along path $i \in \mathrm{P}_{\left\{\mathrm{o}_{g}, \mathrm{~d}_{\mathrm{g}}\right\}}$, with a negative sign. As commonly done in the literature, the weights of the various elements of the generalized travel time are defined relative to the in-vehicle time of the path. We use the values reported in Table 5, obtained from the literature. We assume that passengers have full knowledge of the system and that they choose the path with the highest utility (i.e., the lowest generalized travel time) to travel from origin to destination. Also, our model does not consider demand elasticity, that is, the number of travelers does not change as a consequence of the disruption.

Due to train capacity issues, it is possible that, for some passenger groups, no feasible alternative exists between origin and destination within the time horizon. We therefore include an artificial "penalty path" for those disrupted passenger groups. This path models the worst possible option to travel from origin to destination. We therefore associate it with the lowest possible utility: the duration of the time horizon, with a negative sign.

\subsection{Recovery decisions}

We consider a disruption in the railway network where a number of tracks become unavailable. Multiple track blockages can occur at the same time, and at different locations in the network. We assume that the network is disrupted for the whole time horizon of the rescheduling problem. In order to recover from the disruption, we consider the four following decisions (the three first ones concern original trains):

Cancellation A train may be fully or partially canceled. A partially canceled train is only operated on a subset of the stations of its original route and canceled afterwards. Observe that a full cancellation is a special case of a partial cancellation.

Delay The arrival or departure of a train at a station may be delayed up to a maximal amount of time. A train may also be delayed only for a part of its route. Note that a train with a delay of zero is equivalent to a train in the undisrupted timetable. We do not allow trains to run earlier than in the undisrupted timetable, as this is usually avoided in practice because passengers might miss their planned train.

Rerouting A train may be rerouted through another path than the originally planned one.

Emergency train At every station with a shunting yard, a limited number of emergency trains is available. These may be scheduled as needed. 


\section{Space-time graph}

To represent the problem mathematically, we introduce a directed space-time graph $G(V, A)$, inspired from Nguyen et al. (2001). We first describe the sets of nodes and arcs that characterize all possible movements in the network in the following section. Based on these sets, we describe in Section 4.2 which arcs are available for rescheduling in case of a disruption.

\subsection{Complete graph}

The set of nodes $V=N \cup N_{R} \cup N_{O} \cup N_{D}$ consists of four different types of nodes. A time-expanded node $(s, p, t) \in N$ represents platform $p \in P_{s}$ of station $s \in S$, at time $t \in H$. $N_{R}$ is the set of time-invariant shunting yard nodes, associated with shunting yards $r \in R$. Finally, $N_{O}$ and $N_{D}$ are the sets of time-invariant origin and destination nodes of the passenger groups. We denote by $s(r), s(o)$ and $s(d)$ the station associated with node $r \in N_{R}, o \in N_{O}$ and $d \in N_{D}$, respectively.

Eight types of arcs are defined, representing all feasible movements of trains and passengers:

- Starting arcs model a train leaving the shunting yard at the start of its trip. They are given by the set $A_{\text {Sta }}=\left\{(r,(s, p, t)) \mid r \in N_{R},(s, p, t) \in N, s(r)=s, \forall p \in P_{s}, \forall t \in H\right\}$.

- Ending arcs model a train arriving at the shunting yard at the end of its trip. They are given by the set $A_{\text {End }}=\left\{((s, p, t), r) \mid(s, p, t) \in N, r \in N_{R}, s(r)=s, \forall p \in P_{s}, \forall t \in H\right\}$.

- Driving arcs model the movements of passengers and trains between two neighbouring stations. Train driving arcs are given by the set $A_{\text {Dri }}=\left\{\left((s, p, t),\left(s^{\prime}, p^{\prime}, t^{\prime}\right)\right) \mid(s, p, t),\left(s^{\prime}, p^{\prime}, t^{\prime}\right) \in\right.$ $\left.\mathrm{N}, \forall \mathrm{s}, \mathrm{s}^{\prime} \in \mathrm{S}:\left(\mathrm{s}, \mathrm{s}^{\prime}\right) \in \mathrm{Q}, \forall \mathrm{p} \in \mathrm{P}_{\mathrm{s}}, \forall \mathrm{p}^{\prime} \in \mathrm{P}_{\mathrm{s}^{\prime}}, \forall \mathrm{t}, \mathrm{t}^{\prime} \in \mathrm{H}: \mathrm{t}^{\prime}-\mathrm{t}=\mathrm{t}\left(\mathrm{s}, \mathrm{s}^{\prime}\right)\right\}$. For every passenger group $g$, the set $A_{\text {Dri }}^{g}$ is a duplicate of the arc set $A_{\text {Dri }}$ and represents the driving movements of the passenger group. Arcs in $A_{\text {Dri }}$ and in $A_{\text {Dri }}^{g}$ are weighted differently, as described in Table 2.

- Waiting arcs model passengers and trains waiting in a station. Train waiting arcs are given by the set $A_{W a i}=\left\{\left((s, p, t),\left(s, p, t^{\prime}\right)\right) \mid(s, p, t),\left(s, p, t^{\prime}\right) \in N, \forall s \in S, \forall p \in P_{s}, \forall t, t^{\prime} \in H:\right.$ $t^{\prime}-t=\tau$. Similarly to driving arcs, the set $A_{\text {Wai }}^{g}$ is a duplicate of $A_{\text {Wai }}$, for every passenger group g, and its arcs are weighted accordingly (see Table 2).

- Transfer arcs model passengers transferring from one train to another in a station, with a minimal transfer time $m$ and a maximal transfer time $M$. They are given by the set

$A_{\text {Tra }}^{g}=\left\{\left((s, p, t),\left(s, p^{\prime}, t^{\prime}\right)\right) \mid(s, p, t),\left(s, p^{\prime}, t^{\prime}\right) \in N, \forall s \in S, \forall p \in P_{s}, \forall p^{\prime} \in P_{s} \backslash\{p\}, \forall t, t^{\prime} \in\right.$ $\left.\mathrm{H}: \mathrm{m} \leq \mathrm{t}^{\prime}-\mathrm{t} \leq \mathrm{M}\right\}$.

- Access arcs model passenger group $g$ arriving at the origin. They are given by the set $A_{\text {Acc }}^{g}=\left\{(\mathrm{o},(\mathrm{s}, \mathrm{p}, \mathrm{t})) \mid \mathrm{o} \in \mathrm{N}_{\mathrm{O}},(\mathrm{s}, \mathrm{p}, \mathrm{t}) \in \mathrm{N}, \mathrm{s}(\mathrm{o})=\mathrm{s}=\mathrm{o}_{\mathrm{g}}, \forall \mathrm{p} \in \mathrm{P}_{\mathrm{s}}, \forall \mathrm{t} \in \mathrm{H}\right\}$.

- Egress arcs model passenger group g leaving the system at destination. They are given by the set $A_{\mathrm{Egr}}^{\mathrm{g}}=\left\{((s, \mathrm{p}, \mathrm{t}), \mathrm{d}) \mid(\mathrm{s}, \mathrm{p}, \mathrm{t}) \in \mathrm{N}, \mathrm{d} \in \mathrm{N}_{\mathrm{D}}, \mathrm{s}(\mathrm{d})=\mathrm{s}=\mathrm{d}_{\mathrm{g}}, \forall \mathrm{p} \in \mathrm{P}_{\mathrm{s}}, \forall \mathrm{t} \in \mathrm{H}\right\}$. 
Table 2: Arc weights.

\begin{tabular}{lllll}
\hline Name & Start node & End node & $c_{a}$ & $t_{a}^{g}$ \\
\hline Starting & $r$ & $(s, p, t)$ & 0 & - \\
Ending & $(s, p, t)$ & $r$ & 0 & - \\
Driving & $(s, p, t)$ & $\left(s^{\prime}, p^{\prime}, t^{\prime}\right)$ & $c \cdot d\left(s, s^{\prime}\right)$ & $t\left(s, s^{\prime}\right)$ \\
Waiting & $(s, p, t)$ & $\left(s, p, t^{\prime}\right)$ & 0 & $\beta_{1} \cdot t\left(s, s^{\prime}\right)$ \\
Transfer & $(s, p, t)$ & $\left(s, p^{\prime}, t^{\prime}\right)$ & - & $\beta_{2}+t\left(s, s^{\prime}\right)$ \\
Access & $o$ & $(s, p, t)$ & - & $\beta_{3} \cdot \max \left(0,\left(t_{g}-t\right)\right)+\beta_{4} \cdot \max \left(0,\left(t-t_{g}\right)\right)$ \\
Egress & $(s, p, t)$ & $d$ & - & 0 \\
Penalty & $\mathrm{o}$ & $d$ & - & $n \tau$ \\
\hline
\end{tabular}

- Penalty arcs model passenger group g not succeeding to take the train from origin to destination. They are given by the set $A_{\text {Pen }}^{g}=\left\{(o, d) \mid o \in N_{O}, d \in N_{D}, s(o)=o_{g}, s(d)=d_{g}\right\}$.

The set of train arcs is given by $A_{T}=A_{S t a} \cup A_{E n d} \cup A_{D r i} \cup A_{W a i}$, while the set of passenger arcs associated with passenger group g is $A^{g}=A_{\text {Dri }}^{g} \cup A_{W a i}^{g} \cup A_{\text {Tra }}^{g} \cup A_{\text {Acc }}^{g} \cup A_{\text {Egr }}^{g} \cup A_{\text {Pen }}^{g}$. Note that driving and waiting arcs describe both train and passenger movements. The nodes associated with passenger group $\mathrm{g}$ are denoted by $\mathrm{N}^{\mathrm{g}}$.

The cost of using an arc $a \in A_{T}$ for a train $\left(c_{a}\right)$, or an arc $a \in A^{g}$ for a passenger group $g\left(t_{a}^{g}\right)$, are listed in Table 2. We assume that the operational cost is proportional to the distance travelled by the trains. Therefore, only driving arcs have an operational cost $c_{a}$ different from zero ( $c$ is the cost of running a train, per kilometer). Passenger arcs are weighted according to the utility function introduced in Section 3.2. The cost of a path in the graph for a passenger group is obtained by summing up the weights $t_{a}^{g}$ of the arcs in the path. Note that a driving or waiting arc is weighted differently if is used by a train or a passenger.

\subsection{Rescheduling graph}

The arcs introduced in the previous section represent all possible train and passenger movements in the network. In case of a disruption, some of these movements are forbidden. Also, we need to distinguish train arcs available to original trains and to emergency trains. The general features of the procedure used to generate the rescheduling graph $\mathrm{G}\left(\mathrm{V}, \mathrm{A}^{*}\right)$ are explained here. The interested reader can refer to Appendix A for the detailed algorithm.

When a track becomes unavailable in the network, it cannot be used by any train. The driving arcs in $A_{T}$ corresponding to this track are therefore removed from the graph. We denote by $A^{*} \subset A_{T}$ the subset of available train arcs in the disrupted graph.

The timetable of the original trains is an input to the rescheduling problem. Based on this timetable, we construct, for every original train $k \in K$, the set of available arcs, $A_{k}$, as a subset of the disrupted train arc set, $A^{*}$. An original train can be cancelled, delayed or rerouted. Cancellation is modelled by introducing additional decision variables to the problem (see Section 5). As detailed in Appendix $A$, the sets $A_{k}^{D}$ and $A_{k}^{R R}$ are introduced to model delays and reroutings. For 
every original train $k \in K$, only arcs in $A_{k}=A_{k}^{D} \cup A_{k}^{R R}$ can be used during the disruption. Similarly, we define the set of available nodes, $\mathrm{N}_{k}$, as the set of head nodes of all arcs in $A_{k}$.

By contrast, emergency trains do not have a previous timetable and can therefore be scheduled at any time. The set of available arcs is simply the disrupted train arc set $A^{*}$ in that case. The only constraints for emergency trains is to start and end their trip at a shunting yard and not to use tracks conflicting with other trains. To prevent conflicts, we consider two different situations:

- In the case where all tracks are available, we assume that a separation of $\tau$ between the trains is sufficient. Hence, there is no need to include additional constraints for trains running in the same direction on a track.

- In the case where only one track is available between two neighbouring stations $s, s^{\prime} \in S$, it can be used in both directions. For every train driving arc $a=\left((s, p, t),\left(s^{\prime}, p^{\prime}, t^{\prime}\right)\right) \in A_{\text {Dri }}$, we define the set of conflicting arcs $\Omega(a)=\left\{\left(\left(s_{1}, p_{1}, t_{1}\right),\left(s_{1}^{\prime}, p_{1}^{\prime}, t_{1}^{\prime}\right)\right) \in A_{\text {Dri }} \mid s=s_{1}^{\prime}, s^{\prime}=s_{1}, p=\right.$ $\left.p_{1}, p^{\prime}=p_{1}^{\prime}, t_{1} \geq t, t_{1}<t^{\prime}+\tau\right\}$. This set formalizes the fact that if a train goes from station $s$ to $s^{\prime}$, from time $t$ to $t^{\prime}$, there can be no other train in the opposite direction until time $t^{\prime}+\tau$.

\section{ILP Formulation}

In this section, we present an integer programming formulation for the multi-objective railway timetable rescheduling problem. We consider a tri-objective optimization problem: the quantities to minimize are (i) passenger inconvenience, (ii) operational costs, and (iii) deviation from the undisrupted timetable. The multi-objective aspect of the problem is addressed by using $\varepsilon$-constraints.

Based on the graph defined in the previous section, we introduce the following binary decision variables:

- $w_{\mathrm{a}}^{\mathrm{g}}= \begin{cases}1 & \text { if passenger group } \mathrm{g} \in \mathrm{G} \text { uses arc } \mathrm{a} \in \mathrm{A}^{\mathrm{g}}, \\ 0 & \text { otherwise }\end{cases}$

- $x_{a}^{k}= \begin{cases}1 & \text { if original train } k \in K \text { uses arc } a \in A_{k}, \\ 0 & \text { otherwise }\end{cases}$

- $y_{a}= \begin{cases}1 & \text { if an emergency train uses arc } a \in A^{*} \\ 0 & \text { otherwise }\end{cases}$

- $z_{i}^{k}= \begin{cases}1 & \text { if original train } k \in K \text { is cancelled after node } i \in N_{k} \\ 0 & \text { otherwise }\end{cases}$

Table 3 summarizes all the notations used in the model for the reader's convenience. The last column indicates where the notion is further explained, if necessary. 
Table 3: Notations used in the ILP model.

\begin{tabular}{|c|c|c|}
\hline Name & Description & Reference \\
\hline $\mathrm{G}$ & Set of passenger groups & 3.2 \\
\hline$A^{g}$ & Set of arcs associated with passenger group $g \in G$ & 4.1 \\
\hline $\mathrm{N}^{g}$ & Set of nodes associated with passenger group $\mathrm{g} \in \mathrm{G}$ & 4.1 \\
\hline $\mathrm{N}$ & Set of time-expanded nodes & 4.1 \\
\hline $\mathrm{N}_{\mathrm{R}}$ & Set of shunting yard nodes & 4.1 \\
\hline$S_{R}$ & Set of stations with a shunting yard & 3.1 \\
\hline$A_{i}^{g+} \subset A^{g}$ & Set of passenger arcs leaving node $i \in N \cup N_{O}$ & \\
\hline$A_{i}^{g-} \subset A^{g}$ & Set of passenger arcs entering node $i \in N \cup N_{D}$ & \\
\hline$A_{T}$ & Set of undisrupted train arcs & 4.1 \\
\hline$A^{*} \subset A_{T}$ & Set of disrupted train arcs & 4.2 \\
\hline$A_{i}^{+} \subset A^{*}$ & Set of train arcs leaving node $i \in N \cup N_{R}$ & \\
\hline$A_{i}^{-} \subset A^{*}$ & Set of train arcs entering node $i \in N \cup N_{R}$ & \\
\hline$\Omega(a) \subset A^{*}$ & Set of train arcs conflicting with arc $a \in A^{*}$ & 4.2 \\
\hline K & Set of original trains & 3.1 \\
\hline$A_{k} \subset A^{*}$ & Set of available arcs of original train $k \in K$ & 4.2 \\
\hline $\mathrm{N}_{\mathrm{k}}$ & Set of available nodes of original train $k \in K$ & 4.2 \\
\hline$A_{k}^{\mathrm{D}} \subset A_{k}$ & Set of available delay arcs of original train $k \in K$ & $4.2, \mathrm{~A}$ \\
\hline$A_{k}^{R R} \subset A_{k}$ & Set of available rerouting arcs of original train $k \in K$ & $4.2, \mathrm{~A}$ \\
\hline$A_{k, i}^{+} \subset A_{k}$ & Set of available arcs of train $k \in K$ leaving node $i \in N \cup N_{R}$ & \\
\hline$A_{\mathrm{k}, \mathrm{i}}^{-,} \subset A_{\mathrm{k}}$ & Set of available arcs of train $k \in K$ entering node $i \in N \cup N_{R}$ & \\
\hline$t_{a}^{g}$ & Weight of arc $a \in A^{g}$ used by passenger group $g \in G$ & 4.1 \\
\hline$n_{g}$ & Size of passenger group $g \in G$ & 3.2 \\
\hline$c_{a}$ & Cost of running a train on arc $a \in A_{T}$ & 4.1 \\
\hline$r_{k}$ & Shunting yard node where train $k \in K$ begins its trip & \\
\hline$q$ & Passenger capacity of a train & 3.1 \\
\hline$n_{r}$ & Number of emergency trains available in depot $r \in R$ & 3.1 \\
\hline$t_{i}^{k}$ & $\begin{array}{l}\text { Time difference between node } i \in N_{k} \text { and the original ar- } \\
\text { rival time of train } k \text { at its last station }\end{array}$ & \\
\hline$t_{a}$ & Time duration of arc $a \in A_{k}^{R R}$ & \\
\hline$d_{\mathrm{a}}^{\mathrm{k}}$ & $\begin{array}{l}\text { Delay of arc } a \in A_{k}^{D} \text {, compared to the original timetable of } \\
\text { train } k\end{array}$ & \\
\hline$c_{e}$ & Cost of starting an emergency train & \\
\hline
\end{tabular}




\subsection{Objective functions}

The three objective functions are defined as follows (Eqs. (2)-(4)). As detailed in Section 3.2, passenger inconvenience $\left(z_{p}\right)$ is given by the generalized travel time of the passengers. The operational cost of the timetable $\left(z_{0}\right)$ is the running cost of original trains as well as emergency trains. The deviation $\operatorname{cost}\left(z_{\mathrm{d}}\right)$ represents the deviation from the undisrupted timetable and is a weighted sum of the different rescheduling possibilities: cancellations, reroutings, delays and the cost of adding an emergency train (the respective weighting factors are $\delta_{c}, \delta_{r}, \delta_{d}, \delta_{e}$ ).

$$
\begin{aligned}
z_{p} & =\sum_{g \in G} \sum_{a \in A^{g}} t_{a}^{g} \cdot n_{g} \cdot w_{a}^{g} \\
z_{o} & =\sum_{a \in A^{*}} c_{a} \cdot y_{a}+\sum_{k \in K} \sum_{a \in A_{k}} c_{a} \cdot x_{a}^{k} \\
z_{d} & =\delta_{c} \sum_{k \in K} \sum_{i \in N_{k}} t_{i}^{k} \cdot z_{i}^{k}+\delta_{r} \sum_{k \in K} \sum_{a \in A_{k}^{R R}} t_{a} \cdot x_{a}^{k} \\
& +\delta_{d} \sum_{k \in K} \sum_{a \in A_{k}^{D}} d_{a}^{k} \cdot x_{a}^{k}+\delta_{e} \cdot c_{e} \sum_{r \in R} \sum_{a \in A_{r}^{+}} y_{a}
\end{aligned}
$$

\subsection{Constraints}

The model we propose has three types of constraints: operational constraints, passenger routing constraints and $\varepsilon$-constraints.

Operational constraints Constraints of the first type ensure that all train movements are operationally feasible.

$$
\begin{array}{cr}
\sum_{a \in A_{k, r_{k}}^{+}} x_{a}^{k}=1, & \forall k \in K, \\
\sum_{a \in A_{k, i}^{-}} x_{a}^{k}=\sum_{a \in A_{k, i}^{+}} x_{a}^{k}+z_{i}^{k}, & \forall i \in N_{k}, \forall k \in K, \\
\sum_{i}^{k}=0, & \forall i=(s, p, t) \in N \mid s \notin S_{R}, \\
\sum_{a \in A_{i}^{-}} y_{a}=\sum_{a \in A_{i}^{+}} y_{a}, & \forall r \in N_{R}, \\
\sum_{a \in A_{i}^{-}} y_{a} \leq 1, & \forall i \in N, \\
\sum_{a^{\prime} \in \Omega(a)}\left(y_{a^{\prime}}+\sum_{k \in K} x_{a^{\prime}}^{k}\right) \leq 1, & \forall a \in A^{*} .
\end{array}
$$


Constraints (5) ensure that the original trains leave their shunting yard at the beginning of their trip. Flow conservation constraints (6) make sure that a train either continues its trip after node $i \in N_{k}$, or it is cancelled. Constraints (7) forbid cancellations in stations where there is no shunting yard available. The movements of emergency trains are governed by constraints (8) and (9): there cannot be more trains leaving a shunting yard than the number of trains available in this shunting yard and flow is conserved at every node $i$. Operational conflicts are avoided with constraints (10) and (11). The former ensures that for every node $i$, there is only one incoming emergency train. $\Omega(a)$ is the set of conflicting arcs of $\operatorname{arc} a \in A_{T}$, i.e., if a train is scheduled on arc $a$, there can be no train scheduled on any arc of $\Omega(a)$.

Passenger routing constraints Constraints of the second type deal with the routing of the passengers and are presented below.

$$
\begin{aligned}
& \sum_{\mathrm{a} \in \mathrm{A}_{\mathrm{og}}^{g+}} w_{\mathrm{a}}^{\mathrm{g}}=1, \\
& \sum_{\mathrm{a} \in A_{\mathrm{d}_{g}}^{g-}} w_{\mathrm{a}}^{\mathrm{g}}=1, \\
& \sum_{a \in A_{i}^{g-}} w_{a}^{g}=\sum_{a \in A_{i}^{g+}} w_{a}^{g}, \\
& w_{a}^{g} \leq y_{a}+\sum_{k \in K} x_{a}^{k}, \\
& \sum_{g \in G} w_{a}^{g} \leq q\left(y_{a}+\sum_{k \in K} x_{a}^{k}\right), \\
& \forall \mathrm{g} \in \mathrm{G}, \\
& \forall \mathrm{g} \in \mathrm{G}, \\
& \forall \mathrm{g} \in \mathrm{G}, \forall \mathrm{i} \in \mathrm{N}^{\mathrm{g}}, \\
& \forall \mathrm{g} \in \mathrm{G}, \forall \mathrm{a} \in \mathrm{A}^{*} \cap \mathrm{A}^{\mathrm{g}}, \\
& \forall a \in A^{*} \cap A^{g} .
\end{aligned}
$$

Constraints (12)-(14) are flow conservation constraints for every passenger group: passengers have to leave their origin, arrive at destination and use an uninterrupted path in their respective network. Constraints (15) and (16) link the passenger paths to the train paths and ensure that passengers only use arcs where a train is available, and that train capacities are not exceeded.

$\varepsilon$-constraints To address the multi-objective aspect of the problem, we introduce $\varepsilon$-constraints (see, e.g., Ngatchou et al., 2005). Any of the three objectives can be included as a constraint: $z_{i} \leq \varepsilon_{i}, i \in$ $\{p, o, d\}$. The order in which objectives are minimized as well as the selection process for the upper bounds $\varepsilon_{i}$ is detailed in the next section.

\subsection{Pareto frontier}

The goal of the methodology presented in this section is to explore the three-dimensional Pareto frontier of the problem in an easily interpretable way. By doing so, trade-offs between the three objectives can be quantified in a meaningful way. The generation of the whole exact three-dimensional Pareto frontier (see, e.g., Mavrotas, 2009) is however out of the scope of this work. 
Table 4: Main characteristics of the five-step methodology to explore the Pareto frontier.

\begin{tabular}{|c|c|c|c|c|}
\hline Step & Objective & Constraints & Optimal value & Network \\
\hline (0) & $\min z_{p}$ & $\begin{array}{l}(8)-(16) \\
K=\emptyset\end{array}$ & $z_{\mathrm{p}}^{*}$ & $\mathrm{G}(\mathrm{V}, \mathrm{A})$ \\
\hline$(0 b)$ & $\min z_{\mathrm{o}}$ & $\begin{array}{l}(8)-(16) \\
\mathrm{K}=\emptyset \\
z_{\mathrm{p}}=z_{\mathrm{p}}^{*}\end{array}$ & $z_{\mathrm{o}}^{*}$ & $\mathrm{G}(\mathrm{V}, \mathrm{A})$ \\
\hline (1) & $\min z_{p}$ & $(5)-(16)$ & $z_{\mathfrak{p}}^{* *} \geq z_{\mathfrak{p}}^{*}$ & $\mathrm{G}\left(\mathrm{V}, \mathrm{A}^{*}\right)$ \\
\hline (2) & $\min z_{\mathrm{d}}$ & $(5)-(16)$ & $z_{\mathrm{d}}^{* *}$ & $\mathrm{G}\left(\mathrm{V}, \mathrm{A}^{*}\right)$ \\
\hline & $\min z_{\mathrm{o}}$ & $\begin{array}{l}z_{p} \leq \varepsilon_{p}, \quad \varepsilon_{p}=\varepsilon \cdot z_{p}^{* *} \\
\varepsilon \in\{1.0,1.1, \ldots, 2.0\} \\
(5)-(16)\end{array}$ & & $G\left(V, A^{*}\right)$ \\
\hline (3) & & $\begin{array}{l}z_{\mathrm{p}} \leq \varepsilon_{\mathrm{p}}, \quad \varepsilon_{\mathrm{p}}=\varepsilon \cdot z_{\mathrm{p}}^{* *} \\
z_{\mathrm{d}} \leq \varepsilon_{\mathrm{d}}, \quad \varepsilon_{\mathrm{d}}=\varepsilon \cdot z_{\mathrm{d}}^{* *} \\
\varepsilon \in\{1.0,1.1, \ldots, 2.0\}\end{array}$ & & \\
\hline
\end{tabular}

In order to construct the Pareto frontier, we minimize the objectives in the following order: $z_{\mathrm{p}}$, then $z_{\mathrm{d}}$ (with an upper bound on $z_{\mathrm{p}}$ ), and finally $z_{\mathrm{o}}$ (with upper bounds on $z_{\mathrm{p}}$ and $z_{\mathrm{d}}$ ). Choosing passenger inconvenience as the first objective to minimize seems natural for our passenger-centric formulation. Minimizing the deviation from the undisrupted timetable second is motivated by several computational experiments that showed that the trade-off can be best evaluated if the deviation cost is minimized before the operational cost. The following five-step methodology, summarized in Table 4, is used to explore the Pareto frontier.

The rescheduling problem takes an undisrupted timetable as an input. This timetable needs to be optimal with respect to the objectives we define, so as to have a benchmark - otherwise, the comparison would be unfair. Before solving the problem on the disrupted network $G\left(V, A^{*}\right)$, we therefore solve the problem on the undisrupted network $G(V, A)$, without any original trains, with passenger inconvenience as the objective to minimize. The passenger inconvenience obtained by the first step, $z_{\mathrm{p}}^{*}$, might however be achieved with a lower operational cost, as there is no constraint on the latter. Thus, the next step minimizes the operational cost, while enforcing passenger inconvenience to be equal to the optimal value of the first step. These two steps (denoted by (0) and (0b) in Table 4) thus generate a timetable that is optimal in terms of passenger inconvenience for the undisrupted network, and is associated with the minimal operational cost for that level of inconvenience.

The next step is the first one to be applied on the disrupted network (step (1) of Table 4). As described above, we begin by minimizing the passenger inconvenience, without constraints on operational and deviation cost. This gives an optimal value for the disrupted case, in terms of passenger inconvenience, $z_{\mathfrak{p}}^{* *}$ (that is obviously higher than $z_{\mathrm{p}}^{*}$ ). The objective value of step (1) is then used as an upper bound in the constraints of step (2). The problem is solved with the objective of minimizing $z_{\mathrm{d}}$, under the constraint $z_{p} \leq \varepsilon_{p}, \varepsilon_{p}=\varepsilon \cdot z_{p}^{* *}$. For every value of $\varepsilon \in\{1.0,1.1, \ldots, 2.0\}$, the problem is solved and the value of the optimal deviation cost $z_{\mathrm{d}}^{* *}$ is obtained. This allows to explore the trade-off 
when the passenger inconvenience varies in equally spaced intervals, from the best possible solution $\left(z_{\mathrm{p}}^{* *}\right)$ to a solution with twice the passenger inconvenience. Finally, in the step (3), operational cost is minimized with upper bounds on passenger inconvenience and deviation cost, obtained from steps (1) and (2): $z_{p} \leq \varepsilon_{p}, \varepsilon_{p}=\varepsilon \cdot z_{p}^{* *}$ and $z_{d} \leq \varepsilon_{d}, \varepsilon_{d}=\varepsilon \cdot z_{d}^{* *}$. The problem is solved for every feasible combination of $\varepsilon_{\mathrm{p}}$ and $\varepsilon_{\mathrm{d}}$.

The mathematical model presented in this work is an adaptation from the minimum cost flow problem. Its complexity comes from the routing of the passengers through the network. As the weights of the passenger arcs depend on the passenger group, there needs to be one decision variable per arc and per passenger group. Thus, every additional passenger significantly increases the number of decision variables in the model.

\section{Case study}

We illustrate the methodology on a network constructed from a part of the Dutch railway network. The mathematical model is solved by CPLEX 12.5 on a Unix server with 8 cores of $3.33 \mathrm{GHz}$ and $62 \mathrm{GiB}$ RAM. Most instances are solved to optimality (with a gap of $0.01 \%$ ) in the time limit of one hour.

\subsection{Case description}

We consider the railway network indicated in Figure 1. It represents a heavily used part of the Dutch railway network and consists of 11 stations and 18 tracks between the stations. Each station has two platforms and we consider all track sections to be double tracked. The travel times and distances between the stations are obtained from the Netherlands Railways website. They are reported in Figure 1. We assume there are four stations with shunting yards for original trains (Rot, Ams, Sch, AmZ). Five original trains are located in each of these shunting yards. Furthermore, we assume there are two shunting yards for emergency trains, located in stations Rot and Ams. The number of emergency trains available in each shunting yard is two $\left(n_{r}=2, \forall r \in R\right)$. This gives a total of 24 trains possibly operated in the disrupted case. Every train has a passenger capacity $q$ of 400 . The unit cost $c$ of operating a train in the Netherlands was not available to us. For the sake of this illustrative case study, we have obtained the value from the Swiss Federal Railways annual report (Swiss Federal Railways, 2013), where a regional service costs 30 CHF per kilometer.

We model passengers travelling home in the evening after work and consider a time horizon of two hours, which is discretized into intervals of five minutes. Within this time horizon, passenger groups are generated according to the procedure described in Appendix B. There are 55 passenger groups with a size of 100 passengers each. The generalized travel time of the passengers is computed by using the weights given in Table 5 for the passenger arc costs $t_{\mathrm{a}}^{\mathrm{g}}$. The cost of the penalty arc is the time horizon (two hours). We impose a minimal transfer time $m$ of 5 minutes and a maximal transfer time $M$ of 30 minutes.

Given these inputs, Table 6 indicates the cardinalities of the sets of the space-time graph obtained by following the methodology described in Section 4. 
Table 5: Values of weighting factors in the passengers' generalized travel time.

\begin{tabular}{llll}
\hline Parameter & Value & Unit & Reference \\
\hline$\beta_{1}$ & 2.5 & {$[\mathrm{~min} / \mathrm{min}]$} & Wardman $(2004)$ \\
$\beta_{2}$ & 10 & {$[\mathrm{~min} / \mathrm{transfer}]$} & de Keizer et al. (2012) \\
$\beta_{3}$ & 0.5 & {$[\mathrm{~min} / \mathrm{min}]$} & Small $(1982)$ \\
$\beta_{4}$ & 1 & {$[\mathrm{~min} / \mathrm{min}]$} & Small $(1982)$ \\
\hline
\end{tabular}

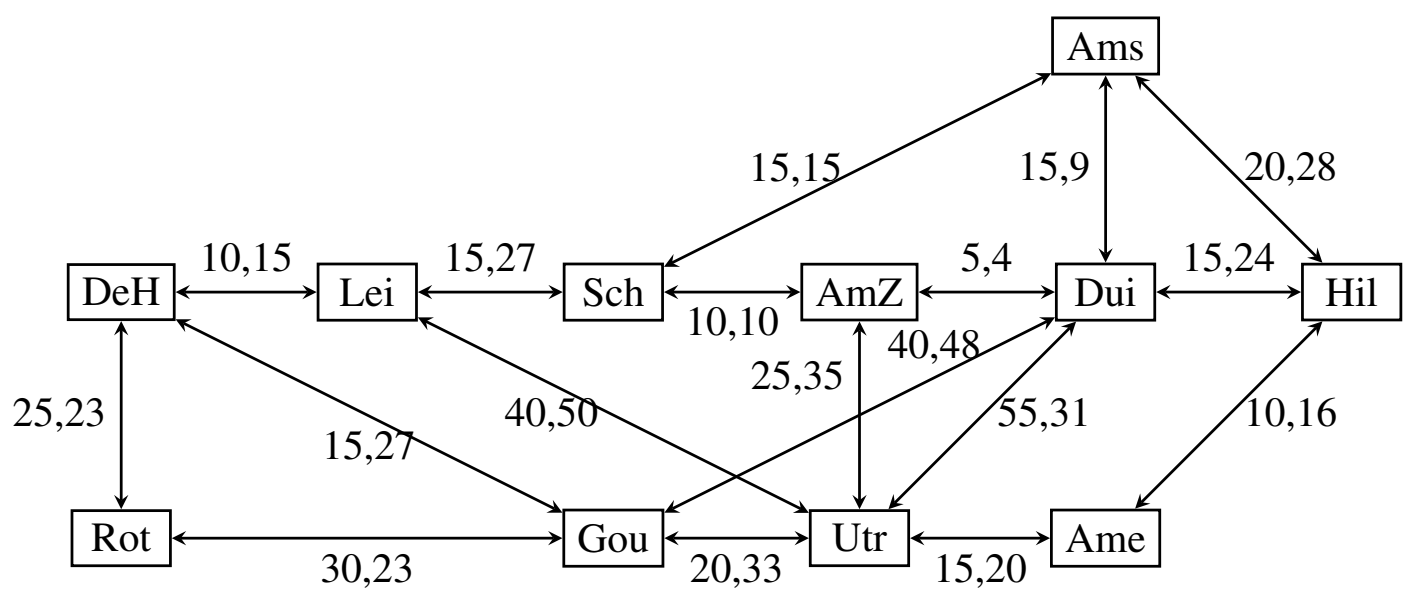

Figure 1: Case study network based on a heavily used part of the Dutch railway network. Stations are indicated by rectangles, and tracks between the stations by double-headed arrows. The first number associated with every track is the travel time between the stations; the second is the travel distance.

Two disruption scenarios are created. For the first disruption scenario, two random tracks are fully blocked. We choose to block the tracks between station Gouda (Gou) and Utrecht (Utr) in both directions. For the second one, we consider the worst-case scenario. To do so, we assume that the most heavily used tracks of the undisrupted timetable are blocked. These turn out to be the tracks between Den Haag (DeH) and Leiden (Lei), and between Leiden (Lei) and Schiphol (Sch). We create the second disruption instance by considering a full blockage of the tracks between theses stations. For both disruption scenarios, we assume the tracks are blocked for the whole time horizon of two hours.

To compute the deviation cost from the undisrupted timetable, we need four different weights: penalties for cancelling, delaying and rerouting trains, and the cost of operating emergency trains. We use the same values as in Veelenturf, Kidd, et al. (2015), where the aim is to operate as many original trains as possible. The cancelled time of a train, i.e., the time difference between the original arrival time of the train at its last station and the time it is cancelled, is weighted heavily by a factor of $\delta_{c}=50$. Every delayed minute, for each train departure, is weighted only by a factor of $\delta_{d}=1$. The time a rerouted train spends on a different geographical path than the original one (i.e., on arcs in $A_{k}^{R R}$ ) is weighted by $\delta_{r}=10$. These values ensure that rerouting a train is preferred over cancelling it, while delaying is the least "costly" option. Finally, the penalty to operate an emergency train $\delta_{e}$ is 
Table 6: Cardinalities of the space-time graph.

\begin{tabular}{cccccc}
\hline Set & Cardinality & Set & Cardinality & Set & Cardinality \\
\hline $\mathrm{N}$ & 550 & $A_{1}$ & 200 & $A_{5}$ & 798 \\
$\mathrm{~N}_{\mathrm{R}}$ & 4 & $A_{2}$ & 200 & $A_{6}$ & 550 \\
$\mathrm{~N}_{\mathrm{O}}$ & 11 & $A_{3}$ & 2992 & $A_{7}$ & 550 \\
$\mathrm{~N}_{\mathrm{D}}$ & 11 & $A_{4}$ & 528 & $A_{8}$ & 110 \\
\hline
\end{tabular}

1,000 plus the operated time of the emergency train. The maximal allowed delay per train $M_{D}$ is set to 30 minutes.

\subsection{Results}

Before applying the rescheduling framework, we run the model on the undisrupted network (i.e., steps $(0)$ and (0b) of Table 4). We obtain a total passenger dissatisfaction of $z_{\mathfrak{p}}^{*}=223^{\prime} 400$ minutes and an operational cost of $z_{\mathrm{o}}^{*}=87^{\prime} 750 \mathrm{CHF}$. In this undisrupted timetable, 20 trains are operated and none of the passenger groups needs to take a penalty arc, as expected.

Tables 7 and 8 show the detailed numerical results for the two disruption scenarios. The two first columns indicate the upper bounds that were imposed on passenger inconvenience and deviation from the undisrupted timetable. The third column gives the optimal value of the minimization of the operational cost (i.e., step (3) of Table 4), and the associated optimality gap is reported in the fourth column. Column give indicated the computational time. Columns six to ten report performance measures of the timetable from the operational point of view: number of rerouted trains, number of totally or partially cancelled trains, number of emergency trains and total delay minutes. Passenger-related performance indicators are reported in columns eleven to fourteen: the average and maximal additional generalized travel time (for non-disrupted passenger groups), and the number of rerouted and disrupted passengers (disrupted passengers use the penalty arc). Also, each block of rows separates instances with different upper bounds on the passenger inconvenience.

For the disruption scenario where tracks between stations Gouda and Utrecht are unavailable, a passenger dissatisfaction of $z_{\mathrm{p}}^{* *}=241^{\prime} 600$ minutes is obtained, when minimizing the latter without any constraints on operational costs and deviation from the undisrupted timetable (step (1) in Table 4). In a second step, the deviation from the undisrupted timetable is minimized, and the optimal value of this problem depends on the bound on the passenger inconvenience (step (2) in Table 4). Table 7 compares a number of solutions of the model, for different values of $\varepsilon_{p}$ and $\varepsilon_{d}$. Note that we only include non-dominated and feasible instances in Table 7.

It can be observed that the number of disrupted passenger groups and the maximal additional travel time strongly depend on the value of the upper bound on the passenger inconvenience, as expected. For an upper bound with $\varepsilon=1$, i.e. $\varepsilon_{\mathrm{d}}=z_{\mathrm{p}}^{* *}=241^{\prime} 600$, there is only one disrupted passenger group and the passenger group whose additional travel time increases most has to travel for 31 minutes more than in the undisrupted timetable. This value of the bound on $z_{p}$ represents the best passenger experience that can be achieved under the circumstances. The price to pay for this level of passenger 
satisfaction is the high operational cost, $96^{\prime} 660 \mathrm{CHF}$ (1.1 times the operational cost of the undisrupted case). However, when $\varepsilon=2$ (i.e., $\varepsilon_{\mathfrak{p}}=2 \cdot z_{\mathfrak{p}}^{* *}$ ), more than half of the passenger groups are disrupted and the additional travel time can be over one and a half hour longer (91 minutes) for the passenger groups who are worst off. This solution is obviously not satisfying from the passenger perspective, but it has a very low operational cost: between 53'160 CHF and 67'700 CHF, depending on the bound on the deviation from the undisrupted timetable. Note that this operational cost is lower than the operational cost of the undisrupted timetable; this is explained by the fact that only trains operated in a timetable account for its operational cost. Hence, if a train is cancelled, the operational cost of the timetable decreases, but it is balanced by an increase of the deviation from the undisrupted timetable. This reasoning also explains why the number of partially cancelled trains increases (from 0 to 4 ) as the operational cost decreases. As cancelling a train is costly (in terms of deviation from the undisrupted timetable), the model will always prefer to cancel a train only partially. Hence, the two trains that are totally cancelled are trains that spent most of their time on the disrupted tracks and therefore need to be cancelled totally. Finally, one can also observe that the number of emergency trains is maximal (4) for the lowest upper bound $\varepsilon_{\mathfrak{p}}$ (thus offering a high level of service to the passengers). This number decreases very quickly when the bound on the passenger inconvenience becomes less tight, as it is very costly (both in terms of operational cost and in terms of deviation from the undisrupted timetable) to schedule an emergency train.

Regarding the disruption scenario where the busiest tracks become unavailable between Den Haag, Leiden and Schiphol, a passenger dissatisfaction of $z_{p}^{* *}=356^{\prime} 700$ minutes is obtained without $\varepsilon$ constraints. The observations that were made for the less severe disruption can be reiterated in this case. The increased severity of the disruption shows in several ways:

- First, the unconstrained value of the passenger dissatisfaction $\left(z_{p}^{* *}\right)$ is almost $60 \%$ higher than in the undisrupted timetable. In other words, in the best case, the passengers will be travelling 1.6 times longer (on average) than in the undisrupted case.

- The upper bounds on the deviation from the undisrupted timetable need to be much higher than in the less severe disruption. Otherwise, the problem becomes infeasible as a too tight bound does not allow enough flexibility in the rescheduling. For instance, the lowest possible bound $\varepsilon_{\mathrm{d}}$ is $17^{\prime} 425$ for disruption DeH-Lei-Sch and 9'590 for disruption Gou-Utr.

- Even in the best possible configuration in terms of passenger dissatisfaction $\left(\varepsilon_{\mathfrak{p}}=z_{\mathrm{p}}^{* *}\right)$, there is a very high number of disrupted passenger groups, and the maximal additional travel time is about one hour and a half.

- The emergency trains are used in many instances, even though they are considered "costly". 


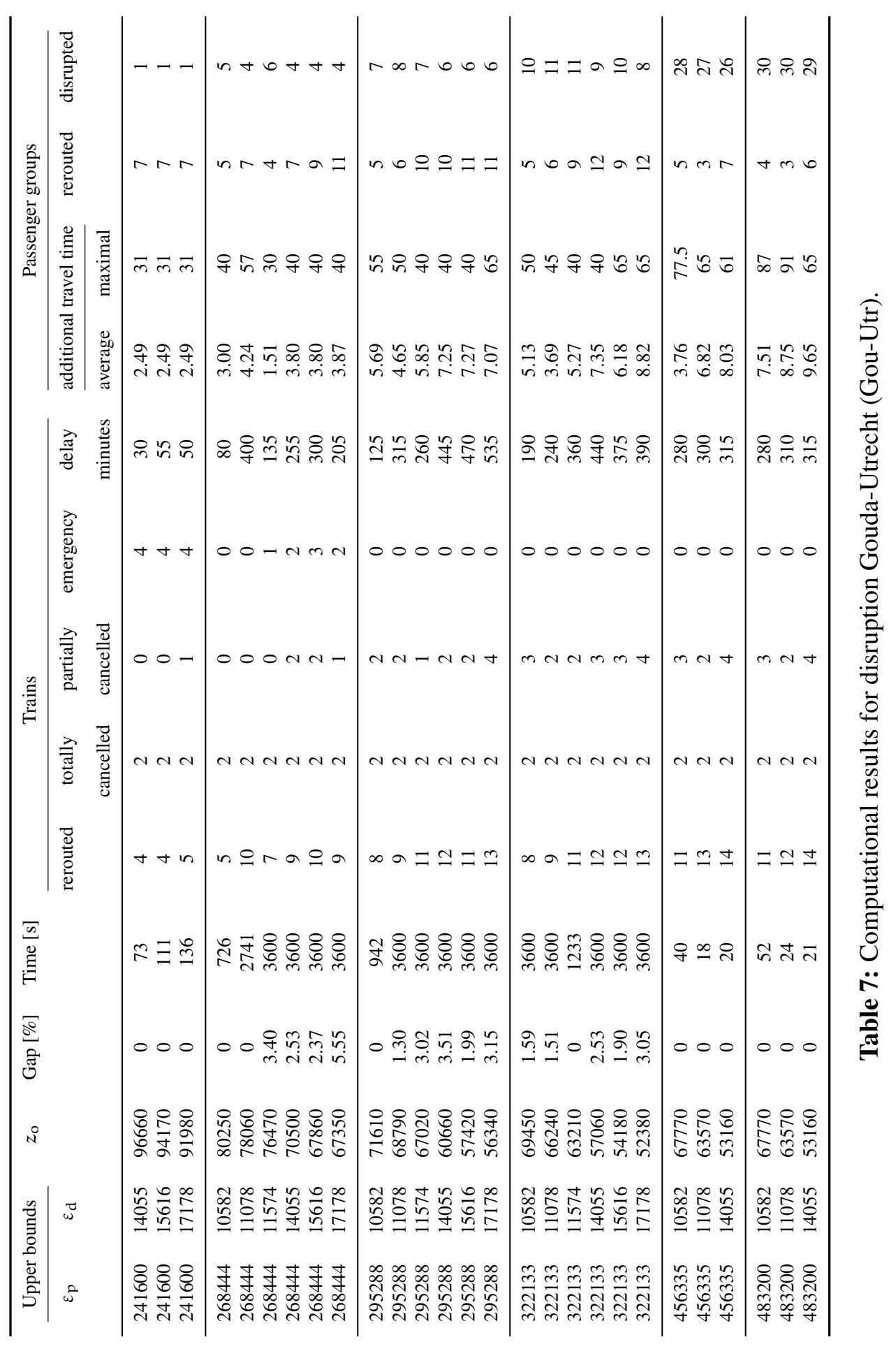




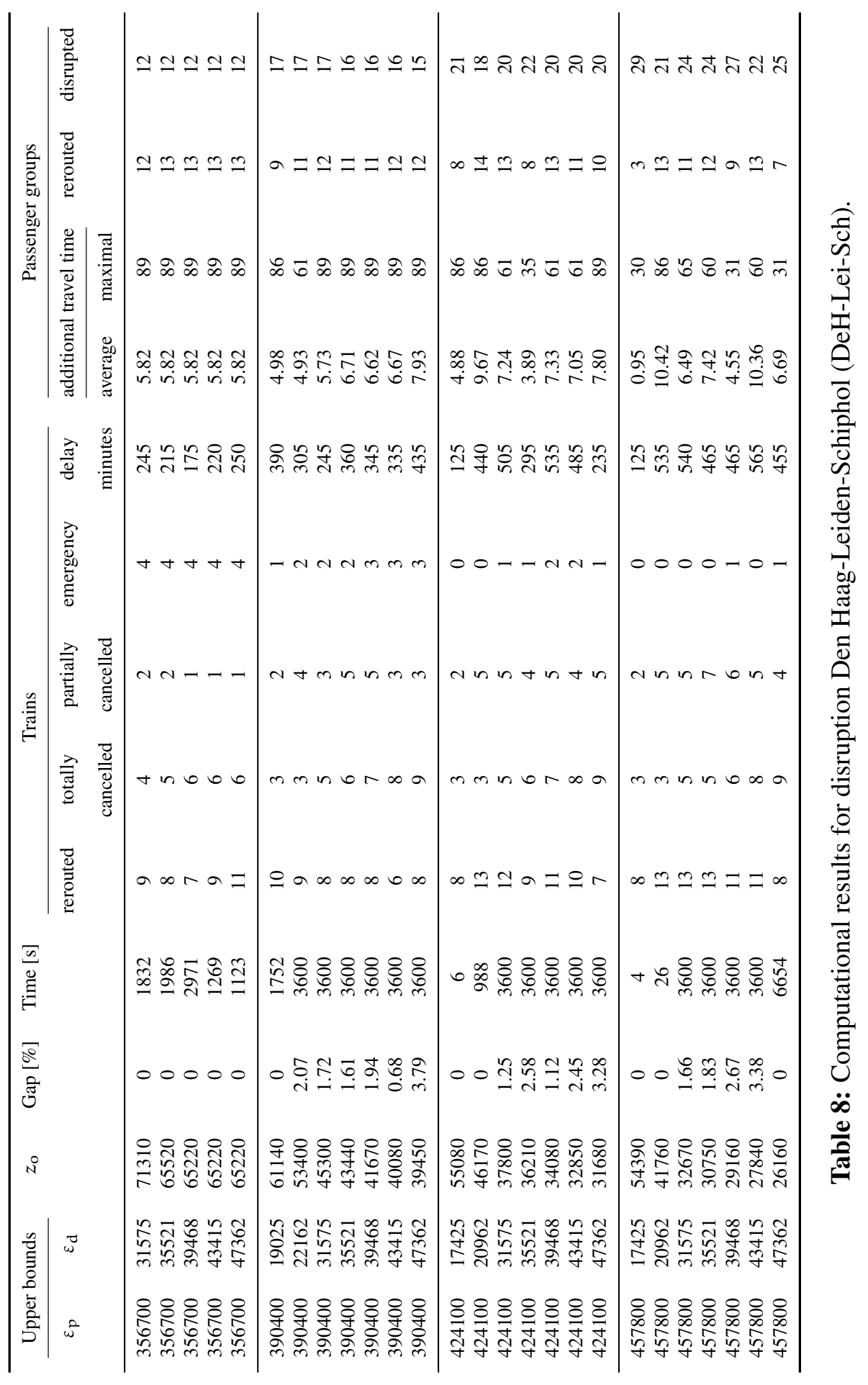




\subsubsection{Pareto frontier}

In order to quantify the trade-off between operational cost and passenger satisfaction, we plot the Pareto frontier for different values of deviation from the undisrupted timetable in Figures $2 \mathrm{a}$ and $2 \mathrm{~b}$. The almost vertical line in Figure $2 \mathrm{a}$ indicates that a significant increase in passenger satisfaction can be achieved with only a little increase in operational cost. We can observe that the higher the deviation from the undisrupted timetable is (i.e., higher $\varepsilon_{\mathrm{d}}$ ), the better the timetable will perform in terms of passenger satisfaction and operational cost. The issue with high values of $\varepsilon_{\mathrm{d}}$ is that the timetable might be very different from the undisrupted one. Hence, the train operating company might want to accept a higher operational cost to achieve the same passenger dissatisfaction, but with a lower deviation from the undisrupted timetable.

Figure $2 \mathrm{~b}$ shows the Pareto frontier for the more severe disruption scenario. Again, the basic observations are consistent between the two plots. The fact that the lines are "less vertical" in the second Figure underlines the severity of the disruption: even by increasing the operational cost, only so much passenger inconvenience decrease can be achieved.

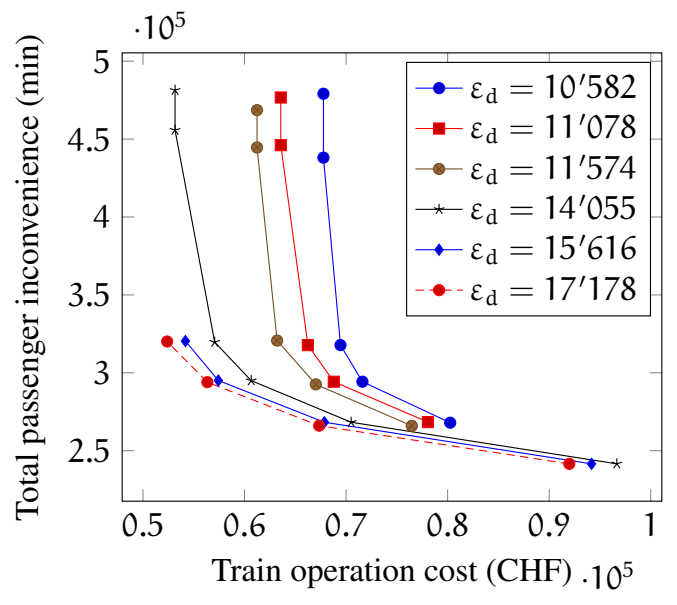

(a) Pareto frontier for disruption Gouda-Utrecht (Gou-Utr).

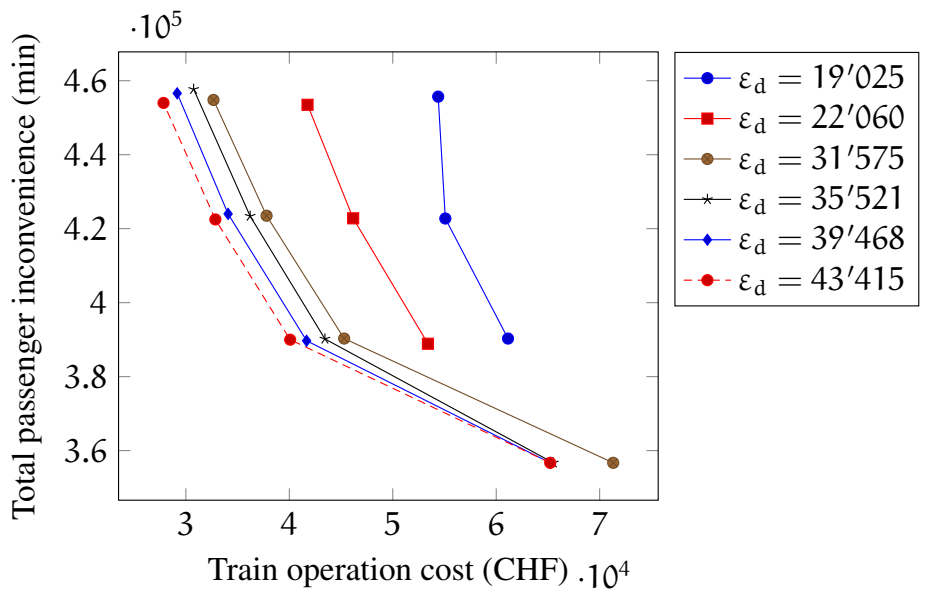

(b) Pareto frontier for disruption Den Haag-LeidenSchiphol (DeH-Lei-Sch).

\section{Conclusion}

In this work, we introduce an integer linear program for the multi-objective railway timetable rescheduling problem for a railway network. We consider passenger inconvenience, operational costs and deviation from the undisrupted timetable as three different objectives to minimize. The infrastructure is modeled from a macroscopic point of view, by considering stations and track sections in between. When a track becomes unavailable, the model choses between delaying, cancelling or rerouting the trains in the undisrupted timetable. The model also includes the possibility to schedule additional emergency trains from depots located near given stations. In addition, passenger flows are adapted dynamically to the new timetable. 
The multi-objective nature of the problem is addressed using epsilon constraints: one objective is minimized while constraints impose an upper bound on the two other ones. This method has the advantage of using meaningful bounds (e.g., the operational cost should not increase by more than $50 \%$ ) in order to construct the Pareto frontier of the problem. This allows to quantify the trade-off between the three conflicting objectives when designing a disposition timetable.

Computational experiments were performed on a case study based on a heavily used part of the Dutch railway network. Results show that significant improvements can be achieved in terms of passenger satisfaction with only a minor increase in the operational cost of the timetable. Also, the higher the deviation from the undisrupted timetable is allowed, the better the timetable will perform in terms of passenger satisfaction and operational cost.

Using a commercial state-of-the-art ILP solver, the model is solved to optimality on most instances, showing that it is possible to account for passenger satisfaction in disposition timetables. Furthermore, it is possible to keep the associated operational costs low and to control for the deviation from the undisrupted timetable. However, the computational time makes the current implementation impractical to use for real-time timetable rescheduling (some instances are solved in about 3 minutes, while others have an optimality gap of $3 \%$ after one hour). It can nonetheless be used by train operating companies that wish to generate offline recovery scenarios for highly disrupted scenarios and quantify their effect regarding the three aforementioned objectives.

The model is a first step towards the integration of passenger-centric indicators in the design of disposition timetables. It is based on several simplifying assumptions. As a follow-up of the proof of concept presented in this paper, several extensions are possible. For instance, we assumed that the passenger demand does not change when the disruption occurs. In reality however, passengers might adjust their destination, their desired departure time, or even their chosen travel mode in a disrupted situation. Hence, an interesting extension would be to account for the shift in the passenger demand following the announcement of the disruption. Also, the iterative combination of an exact operationcentric timetable rescheduling model with a heuristic passenger assignment model could increase the size of solvable instances. Further, our exploration of the Pareto frontier is partial and might miss nondominated solutions. The use of an exact algorithm to explore the three-dimensional Pareto frontier is beyond the scope of this paper, but would definitely be an interesting direction for future research. Finally, since we have ignored the solution methodology side of the problem and rather focused on the general concept, a natural extension would be to aim at a more efficient solving of the problem. We see decomposition methods as one promising option that could allow to solve problems with larger time horizons and, critically, more passengers. 


\section{A Construction algorithm of the rescheduling graph}

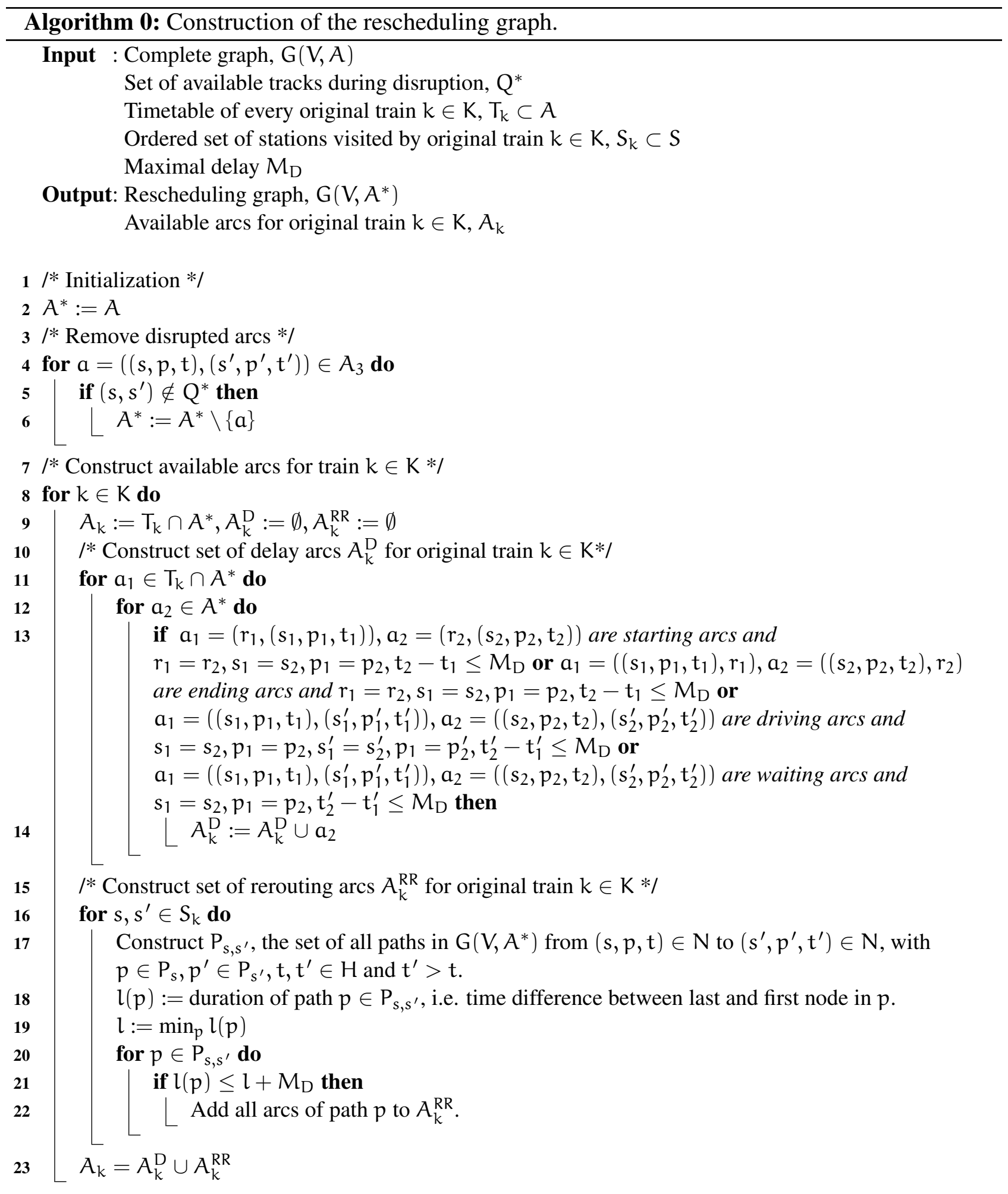




\section{B Passenger demand generation}

Table 9: Probability of a station being chosen as a destination.

\begin{tabular}{lcc}
\hline Station & Probability & Cumulative probability \\
\hline Rotterdam & 0.23 & 0.23 \\
Gouda & 0.03 & 0.26 \\
Utrecht & 0.12 & 0.38 \\
Amersfoort & 0.06 & 0.43 \\
Den Haag & 0.19 & 0.62 \\
Leiden & 0.05 & 0.67 \\
Schiphol & 0.07 & 0.74 \\
Ams. Zuid & 0.07 & 0.82 \\
Duivendrecht & 0.07 & 0.89 \\
Hilversum & 0.03 & 0.93 \\
Amsterdam & 0.07 & 1.00 \\
\hline
\end{tabular}

To model the demand, we define passenger groups characterized by origin station, destination station and desired departure time from origin. As we model people travelling back home in the evening peak hour, we assume that the probability of a station being a destination of a passenger group is proportional to the number of inhabitants in that city. Table 9 shows the probabilities of a station being the destination of a passenger group. The probability of being an origin station is uniformly distributed, with the constraint that the origin station should be different from the destination station. The desired departure time is generated using a non-homogeneous Poisson process: we consider an arrival rate of 50 passenger groups per hour in the first hour and 10 passenger groups per hour in the second. We have a total of 55 passenger groups, where each passenger group has a size of 100 passengers. 


\section{References}

Albrecht, A., D. Panton, and D. Lee (2013). "Rescheduling rail networks with maintenance disruptions using Problem Space Search.” In: Computers \& Operations Research 40.3, pp. 703-712.

Brucker, P., S. Heitmann, and S. Knust (2002). "Scheduling railway traffic at a construction site." In: OR Spectrum 24.1, pp. 19-30.

Cacchiani, V., D. Huisman, M. Kidd, L. Kroon, P. Toth, L. P. Veelenturf, and J. Wagenaar (2014). “An overview of recovery models and algorithms for real-time railway rescheduling." In: Transportation Research Part B: Methodological 63, pp. 15-37.

Cadarso, L., Á. Marín, and G. Maróti (2013). "Recovery of disruptions in rapid transit networks." In: Transportation Research Part E: Logistics and Transportation Review 53, pp. 15-33.

Corman, F., A. D'Ariano, I. A. Hansen, D. Pacciarelli, and M. Pranzo (2011). "Dispatching trains during seriously disrupted traffic situations." In: IEEE International Conference on Networking, Sensing and Control. Delft, pp. 323-328.

Corman, F., A. D’Ariano, A. D. Marra, D. Pacciarelli, and M. Samà (2016). "Integrating train scheduling and delay management in real-time railway traffic control." In: Transportation Research Part E: Logistics and Transportation Review. (Visited on 05/24/2016).

Corman, F., A. D’Ariano, D. Pacciarelli, and M. Pranzo (2014). "Dispatching and coordination in multi-area railway traffic management." In: Computers \& Operations Research 44, pp. 146-160.

De Keizer, B., K. Geurs, and G. Haarsman (2012). "Interchanges in timetable design of railways: A closer look at customer resistance to interchange between trains." In: 40th European Transport Conference. Glasgow.

Dollevoet, T., D. Huisman, M. Schmidt, and A. Schöbel (2012). "Delay Management with Rerouting of Passengers.” In: Transportation Science 46.1, pp. 74-89.

Jespersen-Groth, J., D. Potthoff, J. Clausen, D. Huisman, L. Kroon, G. Maróti, and M. N. Nielsen (2009). "Disruption Management in Passenger Railway Transportation." In: Robust and Online Large-Scale Optimization: Models and Techniques for Transportation Systems. Ed. by R. K. Ahuja, R. H. Möhring, and C. D. Zaroliagis. Berlin, Heidelberg: Springer Berlin Heidelberg, pp. 399-421.

Kroon, L., G. Maróti, and L. Nielsen (2015). "Rescheduling of Railway Rolling Stock with Dynamic Passenger Flows.” In: Transportation Science 49.2, pp. 165-184.

Louwerse, I. and D. Huisman (2014). "Adjusting a railway timetable in case of partial or complete blockades.” In: European Journal of Operational Research 235.3, pp. 583-593.

Mavrotas, G. (2009). "Effective implementation of the epsilon-constraint method in Multi-Objective Mathematical Programming problems.” In: Applied Mathematics and Computation 213.2, pp. 455465.

Narayanaswami, S. and N. Rangaraj (2013). "Modelling disruptions and resolving conflicts optimally in a railway schedule.” In: Computers \& Industrial Engineering 64.1, pp. 469-481.

Ngatchou, P., A. Zarei, and A. El-Sharkawi (2005). "Pareto Multi Objective Optimization.” In: IEEE, pp. 84-91.

Nguyen, S., S. Pallottino, and F. Malucelli (2001). "A Modeling Framework for Passenger Assignment on a Transport Network with Timetables.” In: Transportation Science 35.3, pp. 238-249. 
Robenek, T., Y. Maknoon, S. S. Azadeh, J. Chen, and M. Bierlaire (2016). "Passenger centric train timetabling problem.” In: Transportation Research Part B: Methodological 89, pp. 107-126.

Schachtebeck, M. and A. Schöbel (2010). "To Wait or Not to Wait-And Who Goes First? Delay Management with Priority Decisions.” In: Transportation Science 44.3, pp. 307-321.

Schöbel, A. (2001). "A Model for the Delay Management Problem based on Mixed-Integer-Programming." In: Electronic Notes in Theoretical Computer Science 50.1, pp. 1-10.

- (2009). "Capacity constraints in delay management." In: Public Transport 1.2, pp. 135-154.

Small, K. A. (1982). “The scheduling of consumer activities: Work trips.” In: The American Economic Review 72.3, pp. 467-479.

Swiss Federal Railways (2013). SBB: Facts and Figures 2013.

Veelenturf, L. P., M. P. Kidd, V. Cacchiani, L. Kroon, and P. Toth (2015). "A Railway Timetable Rescheduling Approach for Handling Large-Scale Disruptions." In: Transportation Science.

Veelenturf, L. P., L. Kroon, and G. Maróti (2014). "Passenger Oriented Railway Disruption Management By Adapting Timetables and Rolling Stock Schedules." In: 10th International Conference of the Practice and Theory of Automated Timetabling. PATAT 2014. York, United Kingdom.

Wardman, M. (2004). "Public transport values of time.” In: Transport Policy 11.4, pp. 363-377.

Zhan, S., L. Kroon, L. P. Veelenturf, and J. C. Wagenaar (2015). "Real-time high-speed train rescheduling in case of a complete blockage." In: Transportation Research Part B: Methodological 78, pp. 182-201. 